\title{
Inhibition of 11ß-HSD1, a key enzyme in the stress management, improves cognition by RL-118 drug treatment
}

\author{
Puigoriol-Illamola $\mathrm{D}^{1,2,}$, Vázquez $\mathrm{S}^{3,4}$, Griñán-Ferré $\mathrm{C}^{1,2}$, Pallàs $\mathrm{M}^{1,2}$
}

\begin{abstract}
'Department of Pharmacology, Toxicology and Medicinal Chemistry. Unit of Pharmacology. Faculty of Pharmacy and Food Sciences. University of Barcelona. Av Joan XXIII 27-31, 08028, Barcelona, Spain
\end{abstract}

IInstitute of Neuroscience. University of Barcelona (NeuroUB). Passeig de la Vall d'Hebron 171, Barcelona, Spain

${ }^{3}$ Laboratory of Medicinal Chemistry (Unit associated with Consejo Superior de Investigaciones Científicas). Department of Pharmacology, Toxicology and Medicinal Chemistry. Faculty of Pharmacy and Food Sciences. University of Barcelona. Av Joan XXIII 27-31, 08028, Barcelona, Spain

${ }^{4}$ Institute of Biomedicine. University of Barcelona (IBUB). Barcelona, Spain.

*Author for correspondence: Email: dolo.puigoriol@gmail.com

Received date: November 04, 2020 Accepted date: December 07, 2020

Copyright: @ 2021 Yang H, et al. This is an open-access article distributed under the terms of the Creative Commons Attribution License, which permits unrestricted use, distribution, and reproduction in any medium, provided the original author and source are credited.

Citation: Puigoriol-Illamola D, Vázquez $S$, Griñán-Ferré $C$, Pallàs $M$. Inhibition of $11 \beta$-HSD1, a key enzyme in the stress management, improves cognition by RL-118 drug treatment. Neurosci Chron 2020; 2(1):4-8.
In recent years, stress and stress-coping mechanisms constitute a growing public healthcare issue concerning modern society. Experiencing stress engenders a great complex mechanism named stress response, which consists of a rapid release of catecholamines by the sympathetic nervous system, followed by a slower response in which hormones, mainly glucocorticoids (GCs), are synthesized and released to the bloodstream. Once the stressful stimulus is perceived, the hypothalamus secretes the corticotropin-releasing hormone $(\mathrm{CRH})$, which acts on the pituitary gland, activating the release of adrenocorticotropic hormone (ACTH) that binds to the adrenal glands, promoting GC secretion and conforming the hypothalamus-hypophysis-adrenal (HPA) axis. Under normal conditions, GC secretion follows a robust circadian oscillation with a peak around the onset of the active period of the day, i.e., about 1 hour before arising [1]. This basal level of GC secretion is important in exerting tonic effects upon metabolic, immune and neuronal pathways, involving gluconeogenesis stimulation, protein degradation and lipolysis increase, priming of neural regions involved in sensory processing, attention and adaptive responding, as well as accounting for immunosuppressive and anti-inflammatory actions [2]. However, when stressful exposure is prolonged, the HPA axis deregulates and GC secretion is exacerbated. This excessive GC concentration leads to several metabolic, neurological and behavioral alterations, notably cognitive impairment and affective dysfunctions. GC activity is regulated by $11 \beta$-hydroxysteroid dehydrogenase type 1 (11 $\beta$-HSD1) enzyme, which inhibition has been proved to restore metabolic and behavioral alterations, as well as enhance cognitive abilities. In fact, cortisol, the main active GC in humans, has been postulated as a potential biomarker for neurodegenerative disorders [3], like Alzheimer's disease (AD) in which aging is the major risk factor. Although it is completely assumed that stress directly influences the frailty phenotype in aged people, there are strikingly few measures to restrain stressful lifestyles in order to reduce the progression of pathological towards successful aging. Therefore, the study of stress effects on cognition and its relationship with aging is of the utmost importance to unveil what challenged we might have to cope with as a society in a not so far future.

In consequence, and considering that aging leads to gradual decline of cognitive abilities and is the primary risk factor for $\mathrm{AD}$, we firmly believe that it is necessary taking a step forward in the study of the molecular mechanisms underlying neurodegeneration and find a different approach of the disease to achieve an appropriate treatment. Additionally, it has been described that chronic stress in midlife exerts persisting effects leading to cognitive and affective dysfunctions in old age via mechanisms that depend, at least in part, on brain GCs generated locally by $11 \beta$-HSD1 [4]. Thus, $11 \beta$-HSD1 may be an essential factor in the regulation of the HPA axis and may itself be relevant to age-related diseases susceptibility, severity or outcome [5]. In line with this, elderly who exhibit learning and memory decline showed high GC levels and correlated with greater hippocampal atrophy [6]. Moreover, a recent clinic study published a positive correlation between increased brain $11 \beta$-HSD1 expression with advancing age [7]. Not only in humans, but also in rodents its expression was increased in aged mice and its overexpression accelerated age-related cognitive decline, while 11ß-HSD1-knockout mice resisted age-dependent cognitive loss [8-10].

Another type of chronic stress exposure is metabolic stress induced by a high-fat diet (HFD). In recent years, interest in the impact of nutrition in health has grown since obesity is one of the 
Citation: Puigoriol-Illamola D, Vázquez S, Griñán-Ferré C, Pallàs M. Inhibition of $11 \beta$-HSD1, a key enzyme in the stress management, improves cognition by RL-118 drug treatment. Neurosci Chron 2021; 2(1):4-8.

features of modern society. Mainly, obesity constitutes a risk factor for many disorders, including diabetes, hypertension, cardiovascular alterations, mild cognitive impairment and AD. As mentioned, prolonged GC overexposure has been linked to metabolic disturbances development and 11ß-overexpression in rodents displays a phenotype mimicking human metabolic syndrome, which can be prevented by its inhibition, proposing that intracellular metabolism of GCs by $11 \beta$-HSD1 is critical to the development of insulin resistance rather than the circulating GCs [11-13].

Notwithstanding, it has been suggested a causal role of stress in the onset and progression of age-related cognitive decline and neurodegenerative disorders like $\mathrm{AD}$, as sustained $\mathrm{GC}$ overexposure has been related to amyloid- $\beta$ (A $\beta$ ) formation increase and hyperphosphorylated tau accumulation - hallmarks of $\mathrm{AD}$ and adversely affect behavior [7,14-16]. Accordingly, in clinics, cortisol levels were inversely correlated with cognitive performance and hippocampal volume [17], and a rare single nucleotide polymorphism in the $11 \beta$-HSD1 gene has been reported to increase sporadic AD risk [18], supporting that local tissue levels of GCs may be a significant risk factor for $\mathrm{AD}$ development.

Taking into account all the detrimental effects of excessive GCs and the essential role of $11 \beta-H S D 1$ mediating them, $11 \beta-H S D 1$ inhibitors have been identified and developed. Despite, in the recent years there has been a focus of attention upon targeted 11 $\beta-$ HSD 1 inhibition in the context of metabolic disease, involving insulin resistance and type 2 diabetes mellitus (T2DM), its interest for neurodegenerative diseases treatment has increased. Early clinical studies demonstrated that a $11 \beta$-HSD1 inhibitor (UE2343) is well tolerated and is, therefore, a suitable candidate to improve memory in patients with $\mathrm{AD}[19,20]$. In view of these results, Leiva et al. [21] synthesized a new family of potent $11 \beta$-HSD1 inhibitors, featuring unexplored pyrrolidine-based polycyclic structure. The most potent compounds were characterized in terms of cellular potency, isoenzyme selectivity, human microsomal stability and predicted brain penetration to select a candidate, which was named RL-118 and was selected for in vivo experiments in an animal model of aging, senescence-accelerated mouse prone 8 (SAMP8) (Table 1).

In consequence, we designed and performed different experimental approaches with the aim of determining RL-118 potential beneficial effects in SAMP8 animal, evaluating the underlying mechanisms of RL-118 modulating $11 \beta-H S D 1$ action in different pathways underpinning neurodegeneration, and assessing its role in preventing the negative impact of chronic stress, induced by HFD feeding.

In an attempt to address this issue, different projects were established. In particular, 3 animal studies using SAMP8 mice. On the one hand, although the $11 \beta-H S D 1$ inhibition in the human embryonic kidney (HEK) 293 cells by RL-118 drug was determined, we assessed whether the drug could decrease GCs levels in an animal model. In rodents, the main active GC is corticosterone. Therefore, SAMP8 were divided into 2 groups, control and treated with RL118 , and 2 hours after acute treatment, animals that received RL118 drug showed lower corticosterone levels in blood and brain tissues, so that, effectively, RL-118 was able to cross the blood-brain barrier (BBB) and exert its activity reducing GC action [22].

Afterwards, we designed a study to investigate whether the drug might modulate cognitive abilities to enhance age-related cognitive decline. To address that proof of concept, we used aged SAMP8 animals, divided them into control and under RL118 treatment, treated them for 4 weeks and submitted several behavioral tests. Particularly, we conducted the open field and the object location tests (OFT and OLT, respectively). Behavioral results showed that RL-118 drug decreased recklessness and anxietylike behavior, as well as increased spatial memory, meaning that the drug had a neuroprotective effect. In addition, the mechanisms underlying neuroprotection were studied in the hippocampal tissue. Interestingly, we found that autophagy was promoted with RL-118 treatment, indicating that the drug favored the cellular mechanism to clean harmful material, which positively correlated with behavioral improvement and negatively correlated with pro-inflammatory and oxidative stress (OS) mediators. Not only neuroinflammation and OS were reduced after RL-118 treatment, but also AD hallmarks [23].

Considering the positive effect of the present drug in reverting age-related cognitive decline, our aim was to evaluate in adult mice whether RL-118 had a neuroprotective effect on cognition when animals were exposed to chronic stress situation, represented by HFD-induced metabolic stress approach. 4 groups were established: control fed with normal diet (ND), ND-fed treated with RL-118, HFD-fed and HFD-fed treated with RL-118. Since weaning, mice consumed their dietary condition. At the age of 4 months, drug treatment started for 4 weeks and afterwards, behavioral tests assessing behavioral and cognitive abilities were performed: the three-chamber test (TCT), the novel object recognition test

\begin{tabular}{|c|c|}
\hline Characteristic & RL-118 \\
\hline HEK human HSD ${ }_{1}$ inhibition at $10 \mu M(\%)$ & 100 \\
\hline Human HSD $1 \mathrm{IC}_{50}(\mu \mathrm{M})$ & 0.03 \\
\hline Human $\mathrm{HSD}_{2} \mathrm{IC}_{50}(\mu \mathrm{M})$ & $<0.1$ \\
\hline HLM parent (\%) & 94 \\
\hline PAMPA-BBB $P_{\mathrm{e}}\left(10^{-6} \mathrm{~cm} \mathrm{~s}^{-1}\right)$ & $<30$ (CNS+) \\
\hline
\end{tabular}

Table 1: Biological profile of RL-118 drug. Percentage inhibition was determined compared to a non-inhibitor control. HEK293 cells transfected with the full-length gene coding for human either $11 \beta$-HSD1/2 was used. The microsomal stability was determined using human liver microsomes (HLM) and central nervous system (CNS) + predicted positive blood-brain barrier (BBB) penetration by parallel artificial membrane permeability assay (PAMPA) [21]. 
Citation: Puigoriol-Illamola D, Vázquez S, Griñán-Ferré C, Pallàs M. Inhibition of $11 \beta$-HSD1, a key enzyme in the stress management, improves cognition by RL-118 drug treatment. Neurosci Chron 2021; 2(1):4-8.

(NORT) and the Morris water maze (MWM).

Briefly, our results highlight an improvement in metabolic parameters, such as glucose intolerance and triglyceride concentration, as well as activation of metabolic pathways related to energy sensing like sirtuin 1 (SIRT1)/ peroxisome proliferator-activated receptor gamma coactivator (PGC) $1 \alpha /$ AMP-activated protein kinase $\alpha(\mathrm{AMPK} \alpha)$ signaling route. SIRT1/PGC1 $\alpha / \mathrm{AMPK} \alpha$ axis is regulated by fibroblast growth factor 21 (FGF21) expression. Hormones just as FGF21 and GCs play crucial roles in coordinating the adaptive starvation response. Explicitly, it has been demonstrated that FGF21 modulates energy homeostasis of glucose and lipid through activation of SIRT1/PGC1 $\alpha / \mathrm{AMPK} \alpha$ axis, mainly through liver kinase B1 (LKB1) activation, resulting in enhanced mitochondrial activity [24,25]. In addition, SIRT1 reduces fat accumulation, decreases the risk of visceral obesity and, noteworthy, it has been described that reduces the activity of GC receptors (GR), thus attenuating GCs role in metabolic disorders [26]. Consistently, our results determined that HFD feeding decreased their expression, although treatment with RL-118, thus inhibiting the 11ß-HSD1 enzyme, significantly increased FGF21 and LKB1 protein levels under both dietary conditions $[27,28]$. In line with these results, it has been described that while aging, FGF21 expression increases, but cells apparently become insensitive to it [29]. Herein, we hypothesized that RL-118 treatment could reverse FGF21 resistance, as its protein levels were increased in treated with HFD and RL-118, as well as some of its downstream mediators like AMPK $\alpha$ phosphorylation, suggesting an improvement in nutrient sensing and mitochondrial function. Accordingly, mice that received HFD pronouncedly increased their body weight gain and consumed more calories than ND-fed mice, thus supporting that HFD contributes to glucose tolerance impairment. Despite the studies affirming the use of $11 \beta$-HSD1 inhibitors for the treatment of metabolic syndrome [13] although in accordance with previous results, in our project RL-118 drug did not improve glucose metabolism under normal conditions, though it did in mice receiving concomitant HFD. In addition, these results could be explained by a significant reduction in $11 \beta-H S D 1$ and GR protein levels in the groups that received drug treatment, indicating that when GCs exposure is normalized, metabolic disturbances improve. These results are in line with reports describing a reduction in $11 \beta$-HSD1 and GR gene expression in diet-induced obese mice after treatment with carbenoxolone, a 11ß-HSD1 inhibitor [30].

In agreement with previous projects, OS and neuroinflammation were increased in HFD animals [31] but attenuated after RL-118 treatment. Of note, RL-118 treatment not only decreases ROS concentration, but also favors the ER stress response, which is involved in increasing antioxidant defense mechanisms through the nuclear erythroid-related factor 2 (NRF2). In reference to neuroinflammation, RL-118 drug reduced nuclear factor $\mathrm{kB}$ (NF$\kappa \mathrm{B})$ protein levels, which is involved in pro-inflammatory cytokine expression. Accordingly, pro-inflammatory mediators gene expression was reduced after RL-118 treatment as well as microglia activation, assessed by Iba-1 expression, in the mice hippocampus. Interestingly, $11 \beta$-HSD1 inhibitor treatment enhancement of these markers was notably higher in animals that also received HFD feeding, demonstrating that when SAMP8 mice are under stress conditions, the drug can exert its beneficial effects.

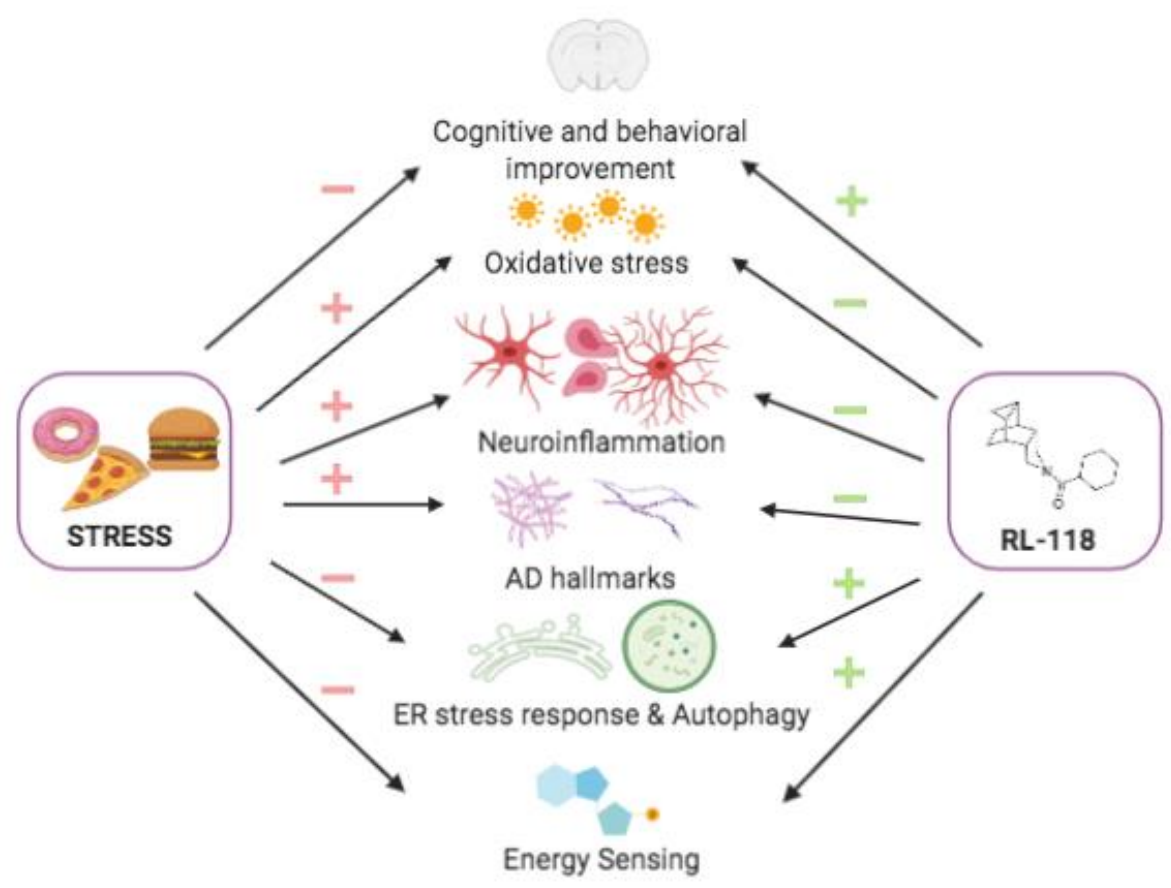

Figure 1: Schematic representation of the molecular pathways affected after HFD feeding and the protective role of RL-118 drug. Chronic stress has an aversive impact on cognitive and behavioral abilities, increases OS and neuroinflammatory markers, promotes AD hallmarks as well as attenuates the UPR and energy-sensing mechanism. By contrast, 11ß-HSD1 inhibition by RL-118 boosts the opposite actions (in red it can be observed the unfavorable effects, whereas in green the beneficial. A positive sign indicates activation or increment, while a negative sign inhibition or decrease, and both signs changes related to both, increases and reductions). 
Citation: Puigoriol-Illamola D, Vázquez S, Griñán-Ferré C, Pallàs M. Inhibition of $11 \beta$-HSD1, a key enzyme in the stress management, improves cognition by RL-118 drug treatment. Neurosci Chron 2021; 2(1):4-8.

Remarkably, drug treatment altered endoplasmic reticulum (ER) stress response in both dietary groups, especially in the HFD mice. In accordance, HFD-fed treated with RL-118 group showed increased Beclin 1 protein levels, thus promoting autophagy [23]. ER stress and autophagy jointly form the unfolded protein response (UPR) to react against misfolded proteins and promote cellular clearance through different mechanisms.

Also, RL-118 treatment induced a reduction of tau hyperphosphorylation and $A \beta$ formation regardless of the diet $[7,23,32]$. In reference to $A D$ hallmarks, not only $A \beta$ formation and tau hyperphosphorylation markers have been evaluated but also others controlling them, like glycogen synthase kinase 3 (GSK3- $\beta)$. It has been reported that GC treatment reduces GSK3$\beta$ expression and function [33]. Consistent with this, it regulates A $\beta$ production by down-regulating the activity of the $\alpha$-secretase and interfering with the $\gamma$-secretase activity, thus resulting in $A \beta$-induced neurotoxicity reduction [34]. Additionally, GSK3- $\beta$ participates in tau phosphorylation and on the contrary, growing evidence indicates that hyperphosphorylated tau activates it, through an increase in OS, neuroinflammation and apoptosis [35]. In consequence, beneficial effects on social behavior and cognitive performance were found in treated mice, supporting the therapeutic strategy that GC excess attenuation by selective $11 \beta$-HSD1 inhibition for the treatment of age-related cognitive decline and $\mathrm{AD}$ through improving metabolic and eventually cognitive disturbances caused by HFD [22].

In conclusion, stress modulates a broad constellation of cellular mechanisms involved in aging and neurodegeneration. Under stressful situations, GCs release is increased, generating an adaptive response. However, when stress is constant, the synthesis and release of GCs, which in physiological conditions is regulated by strict control of the HPA axis, become altered in such a way that large amounts of GCs are released and produce detrimental effects, in particular, on cognition. The metabolic stress induced by HFD has contributed to increasing the deregulation of the HPA axis and thus, GC excess and detrimental molecular mechanisms underpinning neurodegeneration. However, this alteration does not occur only under stressful situations, but also as we age. It is widely recognized that as we age, the body's ability to adapt decreases. Taking into consideration all these reasons, the implication of $11 \beta$-HSD1 in the senescence demonstrated. RL-118 treatment, inhibiting 11ß-HSD1 and therefore reducing GCs exposure, led to cognitive improvement and decreased OS, neuroinflammation and $\mathrm{AD}$ neurodegeneration markers. By contrast, RL-118 increased the UPR response, energysensing mechanisms and synaptic plasticity markers assessed; therefore, providing a protective cellular and effect (Figure 1). Of note, RL-118 treatment was able to restore most of the deleterious effects produced by HFD. Consequently, 11ß-HSD1 could be a feasible target to fight against cognitive decline in age-related pathologies.

\section{References}

1. Son GH, Cha HK, Chung S, Kim K. Multimodal regulation of circadian glucocorticoid rhythm by central and adrenal clocks. Journal of the Endocrine Society. 2018 May;2(5):444-59.

2. Chapman KE, SeckI JR. 11ß-HSD1, inflammation, metabolic disease and age-related cognitive (dys) function. Neurochemical Research. 2008 Apr 1;33(4):624-36.

3. Dhama K, Latheef SK, Dadar M, Samad HA, Munjal A, Khandia R, et al. Molecular Signatures of Biomarkers with a Special Reference to Stress and Related Diseases/Disorders: Diagnostic, Prognostic and Therapeutic Values-Current Progress and Futuristic Vision. Frontiers in Molecular Biosciences. 2019;6:91.

4. Wheelan N, Kenyon CJ, Harris AP, Cairns C, Al Dujaili E, Seckl $\mathrm{JR}$, et al. Midlife stress alters memory and mood-related behaviors in old age: Role of locally activated glucocorticoids. Psychoneuroendocrinology. 2018 Mar 1;89:13-22.

5. Carter RN, Paterson JM, Tworowska U, Stenvers DJ, Mullins JJ, Seckl $J R$, et al. Hypothalamic-Pituitary-Adrenal Axis Abnormalities in Response to Deletion of $11 \beta$-HSD1 is Strain-Dependent. Journal of Neuroendocrinology. 2009 Nov;21(11):879-87.

6. Lara VP, Caramelli P, Teixeira AL, Barbosa MT, Carmona KC, Carvalho $M G$, et al. High cortisol levels are associated with cognitive impairment no-dementia (CIND) and dementia. Clinica Chimica Acta. 2013 Aug 23;423:18-22.

7. Bini J, Bhatt S, Hillmer AT, Gallezot JD, Nabulsi N, Pracitto R, et al. Body Mass Index and Age Effects on Brain 11ß-Hydroxysteroid Dehydrogenase Type 1: a Positron Emission Tomography Study. Molecular Imaging and Biology. 2020 Mar 4:1-8.

8. Caughey S, Harris AP, Seckl JR, Holmes MC, Yau JL. ForebrainSpecific Transgene Rescue of 11ß-HSD1 Associates with Impaired Spatial Memory and Reduced Hippocampal Brain-Derived Neurotrophic Factor mRNA Levels in Aged 11ß-HSD1 Deficient Mice. Journal of Neuroendocrinology. 2017 Jan;29(1).

9. Mohler EG, Browman KE, Roderwald VA, Cronin EA, Markosyan $\mathrm{S}$, Bitner RS, et al. Acute inhibition of $11 \beta$-hydroxysteroid dehydrogenase type-1 improves memory in rodent models of cognition. Journal of Neuroscience. 2011 Apr 6;31(14):5406-13.

10. Yau JL, Noble J, Kenyon CJ, Ludwig M, Seckl JR. Diurnal and stressinduced intra-hippocampal corticosterone rise attenuated in $11 \beta$-HSD1-deficient mice: a microdialysis study in young and aged mice. European Journal of Neuroscience. 2015 Mar;41(6):787-92.

11. Bujalska IJ, Quinkler $M$, Tomlinson JW, Montague CT, Smith DM, Stewart PM. Expression profiling of $11 \beta$-hydroxysteroid dehydrogenase type- 1 and glucocorticoid-target genes in subcutaneous and omental human preadipocytes. Journal of Molecular Endocrinology. 2006 Oct 1;37(2):327-40.

12. Peng $K$, Pan $Y$, Li J, Khan Z, Fan $M$, Yin $H$, et al. $11 \beta$-Hydroxysteroid Dehydrogenase Type 1 (11 $\beta$-HSD1) mediates insulin resistance through JNK activation in adipocytes. Scientific Reports. 2016 Nov $14 ; 6: 37160$.

13. Schnackenberg CG, Costell MH, Krosky DJ, Cui J, Wu CW, Hong VS, et al. Chronic inhibition of $11 \beta$-hydroxysteroid dehydrogenase type 1 activity decreases hypertension, insulin resistance, and hypertriglyceridemia in metabolic syndrome. BioMed Research International. 2013 Jan 1;2013.

14. Bisht K, Sharma K, Tremblay MÈ. Chronic stress as a risk factor for Alzheimer's disease: Roles of microglia-mediated synaptic remodeling, inflammation, and oxidative stress. Neurobiology of Stress. 2018 Nov 1;9:9-21.

15. Green KN, Billings LM, Roozendaal B, McGaugh JL, LaFerla FM. Glucocorticoids increase amyloid- $\beta$ and tau pathology in a mouse model of Alzheimer's disease. Journal of Neuroscience. 2006 Aug 30;26(35):9047-56.

16. MacLullich AM, Ferguson KJ, Reid LM, Deary IJ, Starr JM, Wardlaw $J M$, et al. 11ß-hydroxysteroid dehydrogenase type 1 , brain atrophy and cognitive decline. Neurobiology of Aging. 2012 Jan 1;33(1):207-e1. 
Citation: Puigoriol-Illamola D, Vázquez S, Griñán-Ferré C, Pallàs M. Inhibition of $11 \beta$-HSD1, a key enzyme in the stress management, improves cognition by RL-118 drug treatment. Neurosci Chron 2021; 2(1):4-8.

17. Bloss EB, Morrison JH, McEwen BS. Stress and Aging. The Handbook of Stress. 2011:349-66.

18. de Quervain DJ, Poirier R, Wollmer MA, Grimaldi LM, Tsolaki M, Streffer JR, et al. Glucocorticoid-related genetic susceptibility for Alzheimer's disease. Human Molecular Genetics. 2004 Jan 1;13(1):47-52.

19. Gathercole LL, Lavery GG, Morgan SA, Cooper MS, Sinclair AJ, Tomlinson JW, et al. 11ß-Hydroxysteroid dehydrogenase 1: translational and therapeutic aspects. Endocrine Reviews. 2013 Aug 1;34(4):525-55.

20. Webster SP, McBride A, Binnie M, Sooy K, Seckl JR, Andrew R, et al. Selection and early clinical evaluation of the brain-penetrant $11 \beta$-hydroxysteroid dehydrogenase type $1(11 \beta$-HSD1) inhibitor UE2343 (Xanamem $\left.{ }^{\mathrm{TM}}\right)$. British Journal of Pharmacology. 2017 Mar 1;174(5):396-408

21. Leiva R, Griñan-Ferré $C$, Seira $C$, Valverde E, McBride A, Binnie $M$, et al. Design, synthesis and in vivo study of novel pyrrolidinebased $11 \beta$-HSD1 inhibitors for age-related cognitive dysfunction. European Journal of Medicinal Chemistry. 2017 Oct 20;139:412-28.

22. Puigoriol-Illamola $D$, Leiva $R$, Vázquez-Carrera $M$, Vázquez S, GriñánFerré C, Pallàs M. 11 $\beta$-HSD1 Inhibition Rescues SAMP8 Cognitive Impairment Induced by Metabolic Stress. Molecular Neurobiology. 2020 Jan 1;57(1):551-65.

23. Puigoriol-Illamola $D$, Griñán-Ferré $C$, Vasilopoulou $F$, Leiva $R$, Vázquez S, Pallàs M. 11ß-HSD1 inhibition by RL-118 promotes autophagy and correlates with reduced oxidative stress and inflammation, enhancing cognitive performance in SAMP8 mouse model. Molecular Neurobiology. 2018 Dec 1;55(12):8904-15.

24. Chau MD, Gao J, Yang Q, Wu Z, Gromada J. Fibroblast growth factor 21 regulates energy metabolism by activating the AMPKSIRT1-PGC-1 a pathway. Proceedings of the National Academy of Sciences. 2010 Jul 13;107(28):12553-8.

25. Patel K, Foretz M, Marion A, Campbell DG, Gourlay R, Boudaba N, et al. The LKB1-salt-inducible kinase pathway functions as a key gluconeogenic suppressor in the liver. Nature Communications. 2014 Aug 4;5(1):1-6.

26. Wawryka J, Barg E. Impact of SIRT1 gene expression on the development and treatment of the metabolic syndrome in oncological patients. Pediatric Endocrinology Diabetes and Metabolism. 2016;22(2).

27. Lützner $\mathrm{N}$, Kalbacher $\mathrm{H}$, Krones-Herzig A, Rösl F. FOXO3 is a glucocorticoid receptor target and regulates LKB1 and its own expression based on cellular AMP levels via a positive autoregulatory loop. PloS One. 2012 Jul 27;7(7):e42166.

28. Tezze C, Romanello V, Sandri M. FGF21 as modulator of metabolism in health and disease. Frontiers in Physiology. 2019 Apr 17;10:419.

29. Salminen A, Kaarniranta K, Kauppinen A. Regulation of longevity by FGF21: Interaction between energy metabolism and stress responses. Ageing Research Reviews. 2017 Aug 1;37:79-93.

30. Liu Y, Nakagawa Y, Wang Y, Liu L, Du H, Wang W, et al. Reduction of hepatic glucocorticoid receptor and hexose-6-phosphate dehydrogenase expression ameliorates diet-induced obesity and insulin resistance in mice. Journal of Molecular Endocrinology. 2008 Aug;41(2):53.

31. Palomera-Avalos $V$, Griñán-Ferré $C$, Puigoriol-Ilamola $D$, Camins $A$, Sanfeliu C, Canudas AM, et al. Resveratrol protects SAMP8 brain under metabolic stress: focus on mitochondrial function and Wnt pathway. Molecular Neurobiology. 2017 Apr 1;54(3):1661-76.

32. Canet G, Chevallier N, Zussy C, Desrumaux C, Givalois L. Central role of glucocorticoid receptors in Alzheimer's disease and depression. Frontiers in Neuroscience. 2018 Oct 16;12:739.

33. Llorens-Martın M, Jurado J, Hernández F, Avila J. GSK-3 $\beta$, a pivotal kinase in Alzheimer disease. Front Mol Neurosci. 2014; 7: 46.

34. Saeki K, Machida M, Kinoshita Y, Takasawa R, Tanuma SI. Glycogen synthase kinase-3 $\beta 2$ has lower phosphorylation activity to tau than glycogen synthase kinase-3 $\beta 1$. Biological and Pharmaceutical Bulletin. 2011 Jan 1;34(1):146-9. 